\title{
Malnutrition is Associated with Delayed Sputum Culture Conversion Among Patients Treated for MDR-TB
}

\author{
Asnake Balche Bade' \\ Teshale Ayele Mega (iD ${ }^{2}$ \\ Getandale Zeleke Negera iD $^{3}$ \\ 'School of Pharmacy, Institute of Health \\ Science, Arbaminch University, \\ Arbaminch, Ethiopia; ${ }^{2}$ School of \\ Pharmacy, College of Health Sciences, \\ Addis Ababa University, Addis Ababa, \\ Ethiopia; ${ }^{3}$ Department of Clinical \\ Pharmacy, School of Pharmacy, Institute \\ of Health, Jimma University, Jimma, \\ Oromia Regional State, Ethiopia
}

Background: Clinicians use sputum culture conversion as an interim indicator of the efficacy of multi-drug resistant tuberculosis (MDR-TB) treatment and to determine treatment duration. Yet, limited studies have been published in Ethiopia.

Objective: The objective of this study was to determine the predictors of delayed culture conversion among patients receiving MDR-TB treatment at selected treatment centers in Ethiopia.

Patients and Methods: A multi-center observation study was conducted among MDR-TB patients in South and Southwestern Ethiopia from April 14 to May 14, 2019. The data of patients treated from January 2013 to July 2019 were reviewed using a data abstraction tool. The data were analyzed. Descriptive statistics was computed using SPSS version 21 software program. Cox regression was used to identify predictors of delayed culture conversion. Hazard ratios with a two-sided p-value $<0.05$ were considered statistically significant.

Results: Of 200 included MDR-TB patients, 108 (54\%) were males. Majority,159 (79.5\%) of the patients had a culture conversion time of less than two months, while $15(7.5 \%)$ had delayed culture conversion (greater than 120 days). Patient's registration group (after loss to follow- (adjusted hazard ratio $(\mathrm{AHR})=16.215,95 \%$ CI $[3.839,68.498])$, after treatment failure $(\mathrm{AHR}=12.161,95 \%$ CI $[2.516,58.793])$, history of previous $\mathrm{TB}$ treatment $(\mathrm{AHR}=4.007,95 \%$ CI $[3.115,62.990]))$ and low BMI $(\mathrm{AHR}=1.257 ; 95 \%$ CI $[0.725,1.547]$ were identified as a risk factors for delayed culture conversion.

Conclusion: Our finding showed that nearly $80 \%$ of the patients achieve sputum culture conversion by the second month of treatment. Delayed culture conversion was more likely among patients with malnutrition $\left(\mathrm{BMI}<18.5 \mathrm{~kg} / \mathrm{m}^{2}\right)$, after treatment failure, previous TB treatment, and after lost to follow-up.

Keywords: culture conversion, predictors, MDR-TB, Ethiopia

\section{Introduction}

Tuberculosis (TB) is the commonest infectious disease that is responsible for the death of approximately 2 million people every year, globally. ${ }^{1}$ Although tremendous efforts have been made to control it, an alarmingly increasing Multi-drug resistant tuberculosis (MDR-TB) hampers the prevention and control of $\mathrm{TB}^{2}$ The global TB report showed that in 2018, there were $3.4 \%$ new cases and $18 \%$ previously treated cases of MDR-TB. Countries with the largest share of the global TB burden include the Republic of India, China, and the Russian Federation. In Africa, Nigeria and South Africa harbour the largest number of MDR-TB cases.
Correspondence: Teshale Ayele Mega Addis Ababa University, P.O. Box: 9086, Addis Ababa, Ethiopia

Tel +251913144738

Email tesh.ayu2016@gmail.com 
With 2700 MDR-TB cases each year, Ethiopia is among the top 30 MDR-TB burden countries. A recent data in the country reported the prevalence of MDR-TB to be $0.71 \%$ and $16 \%$ among newly diagnosed and under retreatment, respectively. ${ }^{3}$

The status of mycobacterial cultures plays an important role in guiding the treatment of MDR-TB and is an interim indicator of the efficacy of the treatment. ${ }^{4,5}$ Delayed/nonconversion of sputum culture after the end of 4 months of intensive phase treatment often suggests unfavorable outcomes, ie, treatment failure and death. ${ }^{6,7}$ Furthermore, prolonged sputum culture conversion has a negative impact on the economy, mainly through extending the period of therapy, sub-optimal adherence to treatment with consequent treatment failure. ${ }^{6}$ Baseline radiological findings (lung cavitation), high bacillary load, resistance to ofloxacin (Ofx) or streptomycin, malnutrition, HIV coinfection, and therapeutic delay of greater than one month have been linked to a delay in sputum culture conversion in prior studies. ${ }^{6,8,9}$

Despite the substantial use of sputum culture conversion as an interim indicator of treatment efficacy, limited studies have been conducted in Ethiopia. Hence, this multicenter observational study was designed to determine predictors of delayed sputum culture conversion among patients treated for MDR-TB at three treatment centers in Ethiopia.

\section{Patients and Methods Study Design and Study Area}

A multi-center observational study was done among patients treated for MDR-TB at three treatment centers (Arbaminch, Butajira, and Shenengibe General Hospitals) located in South and Southwestern Ethiopia. The study was conducted from April 14 to May 14, 2019.

Arbaminch and Butajira General Hospitals are located in Southern Ethiopia, and they are currently serving around 11 million population. The former started its MDR-TB treatment service in 2014, while the latter started in 2015. Currently, there are 110 MDR-TB patients registered in both hospitals. Shenengibe Hospital is found in Southwestern Ethiopia, Jimma zone, and it's $329 \mathrm{Km}$ away from the capital, Addis Ababa. It's currently serving about 5 million population. The hospital started it's service in 2013. So far, 98 MDR-TB patients were enrolled in the center.

\section{Study Participants}

We included all adult patients with confirmed diagnosis of MDR-TB (those with isoniazid and rifampicin resistance, but with or without resistance to other first-line drugs) based onXpert MTB/RIF ${ }^{\circledR}$ assay whose charts fulfilled the eligibility criteria (containing complete baseline and follow-up data) in the aforementioned treatment centers. ${ }^{10}$ There is no special sampling technique employed. We excluded charts of patients with incomplete data on the outcome variables, and patients transferred to other treatment centers. Finally, 200 patients were included in the final analysis.

\section{Data Collection}

A structured checklist prepared from different literatures, WHO guidelines, and national MDR-TB treatment followup charts was used to collect the necessary data. The checklist contains several variables. ${ }^{9-12}$ The variables inlude Patient-related variables (gender, age, residence, pregnancy, marital status, smoking status, educational level, and body mass index (BMI)); Disease-related variables (category of MDR-TB, drug resistance status, and comorbidities); Drug-related data (type of medication, and drug regimen). The dependent variable was time to sputum culture conversion.

\section{Definition of Terms}

Sputum culture conversion was defined as a two sets of negative consecutive cultures taken at least 30 days apart after initiation of treatment. Delayed culture conversion was defined as a culture conversion time greater than 120 days. Previously treated: A patient who has a history of previous treatment for TB (four weeks or more), and is now diagnosed with recurrent TB. Treatment after failure: A patient whose treatment was failed at the end of the most recent treatment episode. Treatment after loss to follow up: A patient who has previous history of treatment for $>1$ month and returns to treatment following interruption of treatment for $\geq 2$ consecutive months with positive test by GeneXpert MTB/RIF TB/RIF, culture or sputum smear microscopy. New/previously not treated for TB was defined as a patient who had no prior anti-TB treatment. Adherence was explained as the extent to which a patient continues the agreed upon medication as prescribed (Good Adherence: for adherence rate of $>95 \%$, Fair Adherence: 
adherence rate of $85-94 \%$ and Poor Adherence for $<85 \%$ adherence level).

\section{Data Quality}

The questionnaire was carefully prepared to collect all important data in order to achieve the aim of the study. The appropriateness of each patient chart was reviewed for inclusion before data collection. Two pharmacists and physicians were recruited for data collection. The pharmacists were responsible for collecting drug-related data, while the patientrelated clinical variables were collected by the physicians. At each treatment center, an infectious disease specialist supervised the data collection process. Supervisors followed the data collection process and helped by correlating diagnostic, and laboratory findings with the main outcome. Furthermore, a pretest was done on 5\% (10) of patients' charts to detect the effectiveness of the data collection tool. Based on the pretest finding, all necessary adjustment was made.

\section{Data Analysis}

Data were entered and cleaned in EpiData (v.4.2) and exported to SPSS software package (v.21) for statistical analysis. For categorical variables, proportions were computed and summarized as counts, graphs, and percentages. Pearson's chi- square $\left(\chi^{2}\right)$ was used to compare the baseline characteristics of the patients. For normally distributed variables, mean and standard deviation (SD) were used to summarize, while median and interquartile range (IQR) was utilized to report nonnormally distributed variables. The Kaplan-Meier estimation was performed to compute the baseline survival experience of the patients. A bivariate Cox proportional hazard model was first fitted, and variables with p-value $<0.25$ in the bivariate analysis were further regressed using multivariable Cox proportional-hazard model. On multivariable Cox proportionalhazard regression model, variables with a p-value $<0.05$ were considered to declare statistical significance.

\section{Results}

\section{Overview of the Included Patients}

During the study period, 213 patient charts were reviewed for eligibility and 13 records were excluded. Finally, a total of 200 MDR-TB patients were included in the analysis (Figure 1).

\section{Socio-Demographic Characteristics}

The mean \pm SD age of the patients was $32.9 \pm 9.5$ years. More than half, 108(54\%) of the patients were male and $111(55.5 \%)$ were rural dwellers, and $99(49.5 \%)$ of the

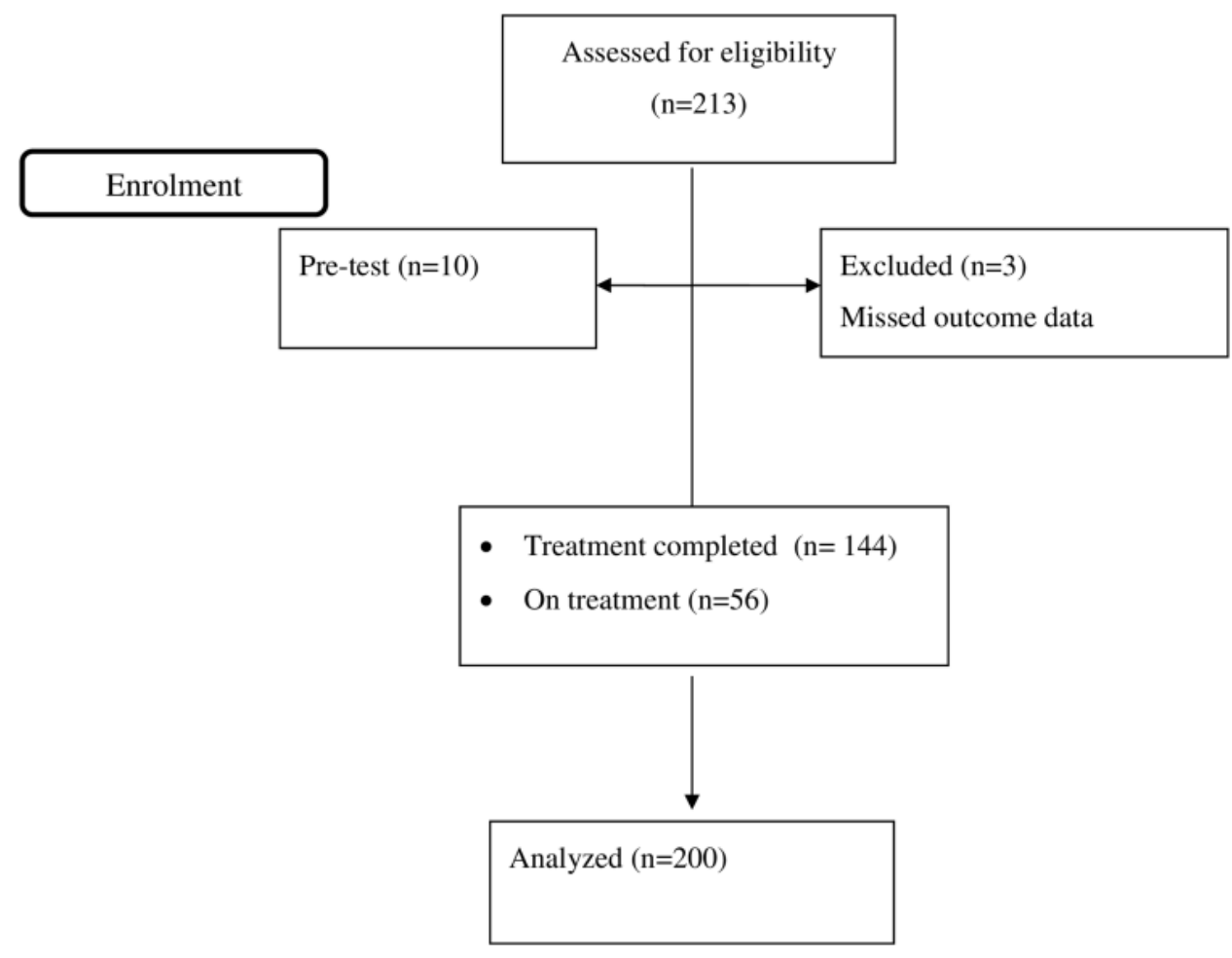

Figure I Flow chart for recruitment of patients treated for MDR-TB treatment, April I4 to May I4, 2019. 
Table I Socio-Demographic Characteristics of the Patients Stratified by the Status of Delayed Culture Conversion Time from April 14 to May 14, 2019

\begin{tabular}{|c|c|c|c|c|}
\hline \multirow[t]{2}{*}{ Variables } & \multirow[t]{2}{*}{ Number of Patients (n) } & \multicolumn{2}{|c|}{ Delayed Culture Conversion } & \multirow[t]{2}{*}{ P-value } \\
\hline & & Yes $(n=15)$ & No $(n=\mid 85)$ & \\
\hline \multicolumn{5}{|l|}{ Sex } \\
\hline Male & $108(54 \%)$ & $8(53.3 \%)$ & 100 (54\%) & 0.957 \\
\hline Female & $92(46 \%)$ & $7(46.7 \%)$ & 85 (46\%) & \\
\hline \multicolumn{5}{|c|}{ Educational level } \\
\hline Primary & $59(29.5 \%)$ & $5(33.3 \%)$ & $54(29 \%)$ & 0.179 \\
\hline Secondary & 74 (37\%) & $7(46.7 \%)$ & $67(36 \%)$ & \\
\hline College & $67(33.5 \%)$ & $3(20 \%)$ & $64(35 \%)$ & \\
\hline \multicolumn{5}{|l|}{ Residence } \\
\hline Urban & $89(44.5)$ & $7(46.7)$ & $82(44.3 \%)$ & 0.861 \\
\hline Rural & III (55.5\%) & $8(53.3 \%)$ & $103(55.7 \%)$ & \\
\hline \multicolumn{5}{|c|}{ Smoking status } \\
\hline Yes & $10(5 \%)$ & $2(13.3 \%)$ & $8(4.3 \%)$ & 0.124 \\
\hline No & 190 (95\%) & $13(86.7 \%)$ & 177 (95.7\%) & \\
\hline \multicolumn{5}{|c|}{ Alcoholic status } \\
\hline Yes & $28(14 \%)$ & I (6.7\%) & $27(14.6 \%)$ & 0.395 \\
\hline No & $172(86 \%)$ & 14 (93.3\%) & $158(85.4 \%)$ & \\
\hline \multicolumn{5}{|c|}{ Marital status } \\
\hline Single & 88 (44\%) & $5(33.3 \%)$ & $82(44.6 \%)$ & 0.791 \\
\hline Married & 99 (49.5\%) & $9(60 \%)$ & 91 (49\%) & \\
\hline Divorce & $10(5 \%)$ & I (6.7\%) & 9 (4.8\%) & \\
\hline Widowed & $3(1.5 \%)$ & $0(0 \%)$ & $3(1.6 \%)$ & \\
\hline
\end{tabular}

patients were married. The highest proportion, 37\%, have a secondary level of education (Table 1).

\section{Clinical Characteristics}

Of the 200 patients, $156(78 \%)$ were previously treated, 22 (11\%) were new MDR-TB cases, 9 (4.5\%) were lost to follow-up, $13(6.5 \%)$ had treatment after failure. About 56 (28\%) of the study participants were living with various comorbidities. The commonest comorbidities diagnosed were diabetes mellitus (DM), 9(4.5\%), and renal problem, 7(3.5\%), respectively. Most, 196 (98\%) of the patients received vitamin B6 supplementation, and 173 (86.5\%) had good adherence (Table 2).

\section{Time to Sputum Culture Conversion}

Majority,159 (79.5\%) of the patients had a culture conversion time of less than two months, while 15 (7.5\%) had delayed culture conversion (greater than 120 days). All patients had converted before 6-months. The median time to culture conversion was 31 (IQR: 30-61) days. There was no statistically significant difference in terms of culture conversion among the regimens ( $\log$ rank $\mathrm{p}=0.53$ ) (Figure 2).

\section{Predictors of Delayed Sputum Culture Conversion}

A Cox proportional-hazard regression analysis was performed to identify predictors of delayed culture conversion. On bivariate analysis, the presence of comorbidity, adherence status, HIV status, BMI, and patient's registration group were significantly associated with delayed culture conversion. On multivariate cox-regression, patient's registration group and BMI remained independent predictors of delayed culture conversion. Consequently, patients registered after loss to follow-up had 16 times higher hazard of delayed culture conversion $(\mathrm{AHR}=16.215,95 \%$ CI [3.839, 68.498]). Similarly, patients who were registered after treatment failure had 12 times higher hazard of delayed culture conversion (AHR $=12.161,95 \%$ CI [2.516, 58.793]). Patients who had history of previous treatment also had 4 times higher hazard of delayed culture 
Table 2 Clinical Characteristics of the Patients Stratified by Delayed Culture Conversion Time from April I4 to May I4, 2019

\begin{tabular}{|c|c|c|c|c|}
\hline \multirow[t]{2}{*}{ Variables } & \multirow[t]{2}{*}{ Number of Patients $(n=200)$} & \multicolumn{2}{|c|}{ Delayed Culture Conversion } & \multirow[t]{2}{*}{ P-value } \\
\hline & & Yes $(n=15)$ & No $(n=185)$ & \\
\hline \multicolumn{5}{|l|}{ Site of disease } \\
\hline Pulmonary & 187 (93.5\%) & $13(86.7 \%)$ & 174 (94\%) & 0.264 \\
\hline Extra-pulmonary & $13(6.5 \%)$ & $2(3.3 \%)$ & II (6\%) & \\
\hline \multicolumn{5}{|l|}{ BMI } \\
\hline$<18.5$ & $55(27.5 \%)$ & II (73.3\%) & $44(23.8 \%)$ & $P<0.01$ \\
\hline$>18.5$ & 145 (72.5\%) & $4(26.7 \%)$ & I4I (76.2\%) & \\
\hline \multicolumn{5}{|l|}{ Registration group } \\
\hline New & 22 (। I\%) & $0(0 \%)$ & 22 (11.9\%) & $P<0.01$ \\
\hline Previously treated & $156(78 \%)$ & $2(13.3 \%)$ & $154(83 \%)$ & \\
\hline After loss to follow-up & $9(4.5 \%)$ & $8(53.3 \%)$ & $\mathrm{I}(0.8 \%)$ & \\
\hline After treatment failure & $13(6.5 \%)$ & $5(33.3 \%)$ & $8(4.3 \%)$ & \\
\hline \multicolumn{5}{|l|}{ Comorbidity } \\
\hline Yes & $56(28 \%)$ & $9(60 \%)$ & 47 (25.4\%) & 0.004 \\
\hline No & 144 (72\%) & $6(40 \%)$ & I 38 (74.6\%) & \\
\hline \multicolumn{5}{|l|}{ HIV } \\
\hline Yes & $44(22 \%)$ & $7(46.7 \%)$ & $37(20 \%)$ & 0.016 \\
\hline No & $156(78 \%)$ & $8(53.3 \%)$ & $148(80 \%)$ & \\
\hline \multicolumn{5}{|l|}{ Renal problem } \\
\hline Yes & 7 (3.5\%) & I (6.7\%) & $6(3.2 \%)$ & 0.488 \\
\hline No & 193 (96.5\%) & 14 (93.3\%) & 179 (96.8\%) & \\
\hline \multicolumn{5}{|l|}{ Diabetes } \\
\hline Yes & $9(4.5 \%)$ & I (6.7\%) & $8(4.3 \%)$ & 0.674 \\
\hline No & 191 (95.5\%) & $14(93.3 \%)$ & 177 (95.7\%) & \\
\hline \multicolumn{5}{|l|}{ Adherence status } \\
\hline Good & 173 (86.5\%) & 5 (33.3\%) & $168(90.8 \%)$ & $\mathrm{P}<0.0 \mathrm{I}$ \\
\hline Fair & $20(10 \%)$ & $8(53.3 \%)$ & $12(6.5 \%)$ & \\
\hline Poor & 7 (3.5\%) & $2(13.4 \%)$ & $5(2.7 \%)$ & \\
\hline \multicolumn{5}{|l|}{ Taking Vit B6 } \\
\hline Yes & 196 (98\%) & $14(93.3 \%)$ & $182(98.4 \%)$ & 0.179 \\
\hline No & $4(2 \%)$ & I (6.7\%) & $3(1.6 \%)$ & \\
\hline \multicolumn{5}{|l|}{ Regimen modified } \\
\hline Yes & $16(8 \%)$ & $2(13.3 \%)$ & 14 (7.5\%) & 0.429 \\
\hline No & $184(92 \%)$ & $13(86.7 \%)$ & $|7|(92.5 \%)$ & \\
\hline
\end{tabular}

Abbreviations: BMI, body mass index; HIV, human immunodeficiency virus; Vit, vitamin.

conversion $\quad(\mathrm{AHR}=4.007,95 \%$ CI $[3.115,62.990])$. Moreover, the hazard of delayed culture conversion among underweight (BMI $<18.5 \mathrm{~kg} / \mathrm{m} 2)$ patients was 1.26 $(\mathrm{AHR}=1.257,95 \%$ CI $[1.125,1.547])$ (Table 3$)$.

\section{Discussion}

In this study, time to sputum culture conversion and predictors of delayed sputum culture conversion were examined. Majority,159 (79.5\%) of the patients had a culture conversion time of less than two months, while 15 (7.5\%) had delayed culture conversion ( $>120$ days). Patient's registration group and BMI were independently associated with delayed culture conversion.

In the present study, $79.5 \%$ of patients had sputum culture converted at 2 months. This finding highlights an effective treatment of the patients with adequate regimens at the right dose, making the patient noninfectious rapidly, which ultimately results in favourable outcomes. Comparable findings 


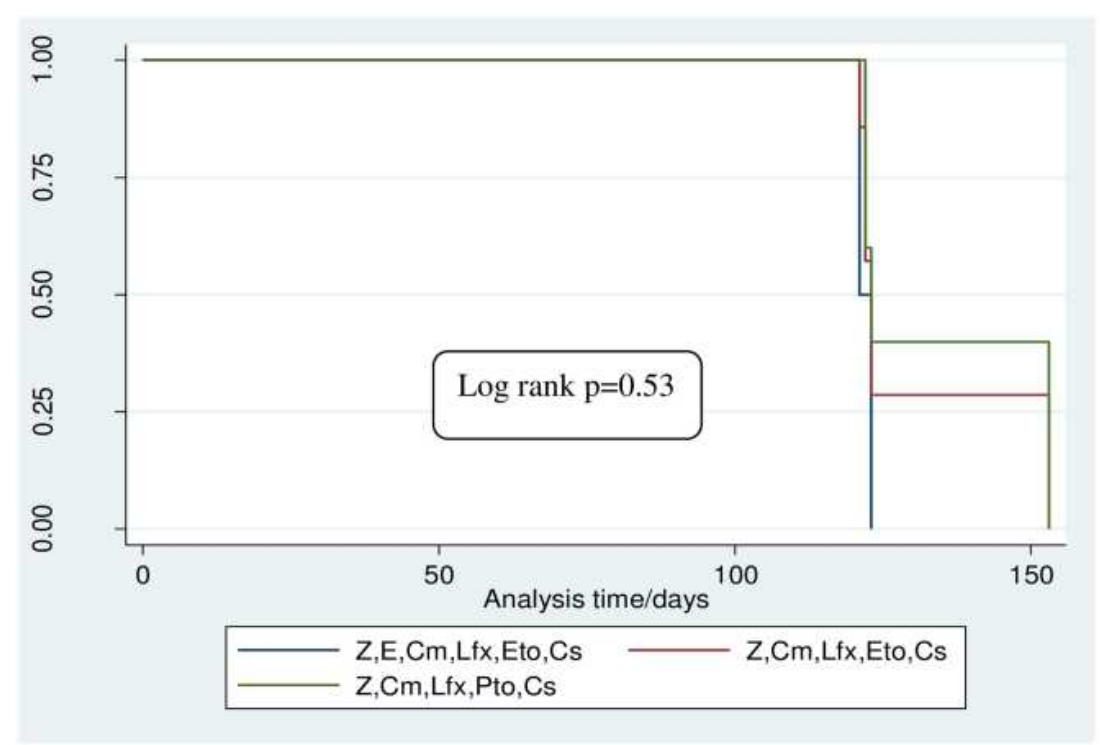

Figure 2 Survival estimates of delayed culture conversion among MDR-TB treated patients January, 2013 to May, 2019.

Abbreviations: Z, pyrazinamide; E, ethambutol; Cm, capreomycin; Lfx, levofloxacin; Eto, ethionamide; Pto, prothionamide; Cs, cycloserine.

have been reported in Latvia ${ }^{13}$ and India, ${ }^{14}$ in which $79 \%$ and $82 \%$ of patients had converted at 2 months, respectively. In contrary, our findings revealed a faster culture conversion in comparison with studies conducted by Kurbatova et $\mathrm{al}^{6}$, and Rodriguez et al. ${ }^{15}$ The variation could be attributed to the difference in sample size and the way time to culture conversion was defined. In this study, time to culture conversion was defined as two consecutive negative culture results, while it was defined by five consecutive negative culture results in the study by Kurbatova et al. ${ }^{6}$

We found that MDR-TB patients with history of previous TB treatment had 4 times higher hazard of delayed culture conversion (AHR $=4.007,95 \%$ CI $[3.115,62.990])$. This finding was in accordance with a study conducted in India, where patients with history of previous TB treatment had two times higher hazard of delayed culture conversion than new MDR-TB patients. ${ }^{16}$

Similarly, patients registered after loss to follow-up (AHR $=16.215,95 \%$ CI $[3.839,68.498])$, and after treatment failure $(\mathrm{AHR}=12.161,95 \% \mathrm{CI}[2.516,58.793])$ also had higher hazard of delayed culture conversion. Similar finding was reported from a multicenter study conducted in Peru, Latvia, Estonia, Russia, and Philippines by Kurbatova et al. ${ }^{6}$

Moreover, MDR-TB patients with low body mass index $\left(\mathrm{BMI}<18.5 \mathrm{~kg} / \mathrm{m}^{2}\right.$ ) had higher hazard of delayed culture conversion than their counterparts $(\mathrm{AHR}=1.257$, $95 \%$ CI $[1.125,1.547])$. This finding was in line with several studies conducted elsewhere that reported low BMI as a predictor of delayed culture conversion. ${ }^{17-19}$ Low BMI is an indicator of poor nutritional status; under nutrition. Under nutrition; an indicator of severe disease and poor socio-economic status, impairs host immunity against mycobacteria. The accompanying impairment in host immunity may increase susceptibility to various infectious diseases. ${ }^{20} \mathrm{~A}$ study by Scrimshaw et $\mathrm{al}^{21}$ particularly revealed that under nutrition (BMI $<18.5 \mathrm{~kg} / \mathrm{m}^{2}$ ) reduced the concentrations of immunoglobulins, interleukin-2 receptors, and T-cell subsets (suppressor-cytotoxic, helper, and natural killer cells) among TB patients. This finding suggests that undernourished MDRTB patients may remain infectious in the community for a long period.

\section{Conclusion}

In conclusion, our finding shows that nearly $80 \%$ of MDRTB patients achieve sputum culture conversion by the second month of treatment. Delayed cultured conversion was more likely in those with malnutrition (BMI $<18.5 \mathrm{~kg} / \mathrm{m}^{2}$ ), after treatment failure, previous TB treatment, and loss to follow-up. 
Table 3 Crude and Adjusted Cox-Proportional Hazard Regression for Predictors of Delayed Culture Conversion of the Cohort, from April 14 to May 14, 2019

\begin{tabular}{|c|c|c|c|c|}
\hline Variables & CHR $[95 \% \mathrm{CI}]$ & P-value & AHR $[95 \% \mathrm{CI}]$ & P-value \\
\hline \multicolumn{5}{|l|}{ Marital status } \\
\hline Single & I.I72[0.370, 3.7|4] & 0.787 & $1.022[0.296,3.534]$ & 0.973 \\
\hline Married & $0.848[0.268,2.88 I]$ & 0.778 & $0.902[0.265,3.074]$ & 0.869 \\
\hline Divorce & $0.609[0.165,2.255]$ & 0.458 & $0.872[0.229,3.324]$ & 0.841 \\
\hline Widowed & 1.00 & & 1.00 & \\
\hline \multicolumn{5}{|l|}{ Comorbidity } \\
\hline Yes & $0.45 \mid[0.319,0.638]$ & $p<0.001$ & $0.739[0.245,2.225]$ & 0.591 \\
\hline No & 1.00 & & 1.00 & \\
\hline \multicolumn{5}{|l|}{ HIV } \\
\hline Yes & $0.509[0.352,0.736]$ & $\mathrm{P}<0.001$ & $0.696[0.245,1.979]$ & 0.497 \\
\hline No & 1.00 & & 1.00 & \\
\hline \multicolumn{5}{|l|}{ DM } \\
\hline Yes & $0.499[0.244,1.021]$ & 0.057 & $0.680[0.238,1.940]$ & 0.470 \\
\hline No & & & 1.00 & \\
\hline \multicolumn{5}{|l|}{ Adherence status } \\
\hline Good & 1.00 & & 1.00 & \\
\hline Fair & $3.004[1.228,7.352]$ & 0.016 & $1.985[0.7 \mid 7,5.497]$ & 0.187 \\
\hline Poor & $0.848[0.299,2.409]$ & 0.757 & $0.994[0.325,3.044]$ & 0.992 \\
\hline \multicolumn{5}{|l|}{ Taking Vit B6 } \\
\hline Yes & 1.00 & 0.103 & 1.00 & 0.769 \\
\hline No & $2.595[0.826,8.154]$ & & $1.220[0.324,4.586]$ & \\
\hline \multicolumn{5}{|l|}{ Regimen modified } \\
\hline Yes & $0.668[0.387, \mathrm{I} .154]$ & 0.148 & $1.220(0.324,4.586)$ & 0.769 \\
\hline No & 1.00 & & 1.00 & \\
\hline \multicolumn{5}{|l|}{ BMI } \\
\hline$<18.5$ & $0.76 \mid[0.64 \mid, 0.903]$ & 0.002 & $1.257[1.125,1.547]$ & 0.046 \\
\hline$>18.5$ & 1.00 & & 1.00 & \\
\hline \multicolumn{5}{|l|}{ Hgb } \\
\hline Low HGB & $0.743[0.494,1.116]$ & 0.152 & $0.820[0.324,2.075]$ & 0.676 \\
\hline Normal HGB & 1.00 & & 1.00 & \\
\hline \multicolumn{5}{|l|}{ Registration group } \\
\hline New & 1.00 & - & 1.00 & - \\
\hline Previously treated & $7.031[3.980,71.889]$ & $P<0.001$ & $4.007[3.115,62.990]$ & 0.001 \\
\hline After loss to follow-up & $18.210[4.475,74.099]$ & $p<0.001$ & $16.215[3.839,68.498]$ & $P<0.001$ \\
\hline After treatment failure & $|2.89|[2.776,59.866]$ & 0.001 & $12.16 \mid[2.5 \mid 6,58.793]$ & 0.002 \\
\hline
\end{tabular}

Abbreviations: BMI, body mass index; DM, diabetes mellitus; Hgb, haemoglobin; Vit, Vitamin.

\section{Limitations of the Study}

This study suffers from several limitations. Firstly, the retrospective nature of the data source limited us from capturing full information about the patients. Secondly, some important diagnostic and laboratory data were missed due to lack of sufficient registration. Hence, most of the patients have no data on sputum smear microscopy results. Thirdly, the method of patients' adherence assessment was also subjective as it is based on patient reports. Lastly, although his was a multi-center survey, the number of hospitals included in the study was not sufficient to fully capture the data in Ethiopia. 


\section{Abbreviations}

BMI, body mass index; Cm, capreomycin; E, ethambutol; Eto, ethionamide; MDR-TB, multidrug resistance tuberculosis; PZA, pyrazinamide; $\mathrm{R}$, rifampici.

\section{Data Sharing Statement}

There are no material outputs from this study, and all data are those presented in the manuscript.

\section{Ethical Approval}

The study approved by the Ethical Review Board of Jimma University and given an IRB number of IHRPG1/ $565 / 2019$. Because of the retrospective anonymous nature of the study, the need for informed consent was waived. Confidentiality was ensured by removing the name and address of the patients from the data. This study was conducted in accordance with the Declaration of Helsinki.

\section{Acknowledgments}

The authors thank the data collectors and all staff members of the study settings for their valuable contribution. We also would like to thank Jimma University for providing this opportunity to conduct this research.

\section{Author Contributions}

All authors made a significant contribution to the work reported, whether that is in the conception, study design, execution, acquisition of data, analysis and interpretation, or in all these areas; took part in drafting, revising or critically reviewing the article; gave final approval of the version to be published; have agreed on the journal to which the article has been submitted; and agree to be accountable for all aspects of the work. All authors have read and approved the manuscript.

\section{Funding}

The authors received no specific funding for this work.

\section{Disclosure}

The authors declare no conflicts of interest for this work.

\section{References}

1. World Health Organization. Global tuberculosis report. Geneva: World Health Organization. 2019; Available from: https://www.who.int/tb/ publications/global report/en/. Accessed April 13, 2021.

2. Ormerod LP. Multidrug-resistant tuberculosis (MDR-TB): epidemiology, prevention and treatment. Br Med Bull. 2005;73-74(1):17-24. doi: $10.1093 / \mathrm{bmb} / \mathrm{ldh} 047$
3. Ismail $\mathrm{N}$, Ismail $\mathrm{F}$, Omar $\mathrm{SV}$, et al. Drug resistant tuberculosis in Africa: current status, gaps and opportunities. 2016;7(2):1-11. Afr $J$ Lab Med. 2018;7(2):1-11. doi:10.4102/ajlm.v7i2.781

4. Laserson KF, Thorpe LE, Leimane V, et al. Speaking the same language: treatment outcome definitions for multi drug-resistant tuberculosis. Int J Tuberc Lung Dis. 2005;9(6):640-645.

5. World Health Organization. Guidelines for establishing DOTS-plus pilot projects for the management of Multidrug-Resistant Tuberculosis (MDR-TB). WHO/CDS/TB/2000.279. Geneva: World Health Organization; 2000

6. Kurbatova E, Gammino V, Bayona J, et al. Predictors of sputum culture conversion among patients treated for multidrug-resistant tuberculosis. Int $J$ Tuberc Lung Dis. 2012;6(10):1335-1343. doi:10.5588/ijtld.11.0811

7. Kurbatova EV, Cegielski JP, Lienhardt C, et al. Sputum culture conversion as a prognostic marker for end-of-treatment outcome in patients with multidrug-resistant tuberculosis: a secondary analysis of data from two observational cohort studies. Lancet Respir Med. 2015;3(3):201-209. doi:10.1016/S2213-2600(15)00036-3

8. Basit A, Ahmad N, Khan AH, et al. Predictors of two months culture conversion in multidrug-resistant tuberculosis: findings from a retrospective cohort study. PLoS One. 2014;9(4):e93206. doi:10.1371/journal.pone.0093206

9. Putri FA, Burhan E, Nawas A, et al. Body mass index predictive of sputum culture conversion among MDR-TB patients in Indonesia. Int $J$ Tuberc Lung Dis. 2014;18(5):564-570. doi:10.5588/ ijtld.13.0602

10. Alene K, Viney K, Mcbryde S, Tsegaye A. Treatment outcomes in patients with multidrug-resistant tuberculosis in Treatment outcomes in patients with multidrug-resistant tuberculosis in north-west Ethiopia. Trop Dis Med Vaccine. 2017;22(3):351-362.

11. Caminero JA Guidelines for clinical and operational management of drug-resistant tuberculosis; 2013.

12. Caminero JA. State of the ART multidrug-resistant tuberculosis: epidemiology, risk factors, and case finding. Int J Tuberc Lung Dis. 2010;14(4):382-390.

13. Holtz TH, Sternberg M, Kammerer S, et al. Time to sputum culture conversion in multidrug-resistant tuberculosis: predictors and relationship to treatment outcome. Ann Intern Med. 2006;144 (9):650-659. doi:10.7326/0003-4819-144-9-200605020-00008

14. Joseph P, Desai VB, Mohan NS, et al. Outcome of standardized treatment for patients with MDR-TB from Tamil Nadu, India. Indian J Med Res. 2011;133(5):529-534.

15. Rodriguez M, Monedero I, Caminero JA, et al. Successful management of multidrug-resistant tuberculosis under programme conditions in the Dominican Republic. Int J Tuberc Lung Dis. 2013;17 (4):520-525. doi:10.5588/ijtld.12.0481

16. Katiyar SK, Bihari S, Prakash S, Mamtani M, Kulkarni H. A randomised controlled trial of high-dose isoniazid adjuvant therapy for multidrug-resistant tuberculosis. Int J Tuberc Lung Dis. 2008;12 (2):139-145.

17. Park HO, Kim SH, Moon SH, et al. Association between body mass index and sputum culture conversion among South Korean patients with multidrug resistant tuberculosis in a tuberculosis referral hospital. Infect Chemother. 2016;48(4):317-323. doi:10.3947/ ic.2016.48.4.317

18. Velayutham B, Nair D, Kannan T, et al. Factors associated with sputum culture conversion in multidrug-resistant pulmonary tuberculosis. Int $J$ Tuberc Lung Dis. 2016;20(12):1671-1676. doi:10.5588/ijtld.16.0096

19. Magee MJ, Kempker RR, Kipiani M, et al. Diabetes mellitus, smoking status, and rate of sputum culture conversion in patients with multidrug-resistant tuberculosis: a cohort study from the country of Georgia. PLoS One. 2014;9(4):e94890. doi:10.1371/journal. pone. 0094890 
20. Lee YM, Kim SM, Park SJ. Factors associated with a strong response to the T-SPOT. TB in patients with extrapulmonary tuberculosis. Infect Chemother. 2014;46(4):248-252. doi:10.3947/ic.2014.46.4.248
21. Scrimshaw NS, SanGiovanni JP. Synergism of nutrition, infection, and immunity: an overview. Am J Clin Nutr. 1997;66(2):464S-477S. doi:10.1093/ajen/66.2.464S

\section{Publish your work in this journal}

Infection and Drug Resistance is an international, peer-reviewed openaccess journal that focuses on the optimal treatment of infection (bacterial, fungal and viral) and the development and institution of preventive strategies to minimize the development and spread of resistance. The journal is specifically concerned with the epidemiology of

Submit your manuscript here: https://www.dovepress.com/infection-and-drug-resistance-journal antibiotic resistance and the mechanisms of resistance development and diffusion in both hospitals and the community. The manuscript management system is completely online and includes a very quick and fair peerreview system, which is all easy to use. Visit http://www.dovepress.com/ testimonials.php to read real quotes from published authors. 